\title{
Small aspect ratio Taylor-Couette flow: Onset of a very-low-frequency three-torus state
}

\author{
Juan M. Lopez* \\ Department of Mathematics and Statistics, Arizona State University, Tempe, Arizona 85287-1804, USA \\ Francisco Marques ${ }^{\dagger}$ \\ Departament de Física Aplicada, Universitat Politècnica de Catalunya, Jordi Girona Salgado s/n, Mòdul B4 Campus Nord, \\ 08034 Barcelona, Spain
}

(Received 7 January 2003; published 3 September 2003)

\begin{abstract}
The nonlinear dynamics of Taylor-Couette flow in a small aspect ratio annulus (where the length of the cylinders is half of the annular gap between them) is investigated by numerically solving the full threedimensional Navier-Stokes equations. The system is invariant to arbitrary rotations about the annulus axis and to a reflection about the annulus half-height, so that the symmetry group is $\operatorname{SO}(2) \times Z_{2}$. In this paper, we systematically investigate primary and subsequent bifurcations of the basic state, concentrating on a parameter regime where the basic state becomes unstable via Hopf bifurcations. We derive the four distinct cases for the symmetries of the bifurcated orbit, and numerically find two of these. In the parameter regime considered, we also locate the codimension-two double Hopf bifurcation where these two Hopf bifurcations coincide. Secondary Hopf bifurcations (Neimark-Sacker bifurcations), leading to modulated rotating waves, are subsequently found and a saddle-node-infinite-period bifurcation between a stable (node) and an unstable (saddle) modulated rotating wave is located, which gives rise to a very-low-frequency three-torus. This paper provides the computed example of such a state, along with a comprehensive bifurcation sequence leading to its onset.
\end{abstract}

DOI: 10.1103/PhysRevE.68.036302

PACS number(s): 47.20.Ky, 47.15.Fe, 47.54.+r, 47.32.-y

\section{INTRODUCTION}

Taylor-Couette flow between two concentric rotating cylinders continues to provide a canonical physical system that has been instrumental in developments in nonlinear dynamics, routes to chaos, and equivariant dynamical systems (e.g., Refs. [1-3]). The study of the influence of endwalls and reflection symmetry at the annulus half height $[4,5]$ opened up a new perspective into the importance of $Z_{2}$ symmetry (reflection) in Taylor-Couette flow, as well as in many other equivariant problems.

The impact of $Z_{2}$ symmetry is enhanced as the aspect ratio of the annulus is reduced [6]. Most theoretical and numerical studies of short annulus Taylor-Couette flow have been restricted to an axisymmetric subspace and have primarily considered steady $Z_{2}$-symmetry breaking. Experimentally however, very rich and complex spatiotemporal dynamics, including global bifurcations, have been observed, which are intrinsically associated with the $Z_{2}$ equivariance of the system (e.g., Refs. [7-10]). Equivariant bifurcation theory [11-13] provides a classification of possible bifurcation scenarios that may occur in the presence of symmetries. Two recent studies on short annulus Taylor-Couette flow with $Z_{2}$ reflection symmetry have identified two of the ways the basic state can become unstable, both breaking the $Z_{2}$ symmetry; in Ref. [14] the symmetry breaking is via steady-state pitchfork bifurcations and in Ref. [15] it is via a Hopf bifurcation. In both investigations, the analyses of the results were restricted to an axisymmetric $\mathrm{SO}(2)$ subspace.

One of the main conclusions from Ref. [14] is the conjec-

\footnotetext{
*Electronic address: lopez@math.asu.edu

${ }^{\dagger}$ Electronic address: marques@fa.upc.es
}

ture that at small aspect ratios (less than 0.5), the steady axisymmetric flow is unique, and yet they report observing time dependence and disorder at higher Reynolds number Re and claim that an understanding of the evolution remains an outstanding challenge. In this paper we investigate numerically the dynamics at aspect ratio $\Gamma=0.5$ using a threedimensional Navier-Stokes spectral solver. At small Re we found the steady-state pitchfork bifurcation that breaks the $Z_{2}$ symmetry, as well as the inverse pitchfork where the bifurcated states revert to a $Z_{2}$-symmetric state, as reported in Ref. [14]. At slightly higher Re, the flow becomes unstable via two Hopf bifurcations that break $Z_{2}$, one leading to an axisymmetric limit cycle and the other to a nonaxisymmetric rotating wave. In fact, we have located a codimension-two bifurcation point where both Hopf bifurcations occur simultaneously. By carefully following the various bifurcated states, we have constructed a fairly complete bifurcation picture that culminates in the onset of a very-lowfrequency three-torus state via a saddle-node-infinite-period global bifurcation.

\section{BASIC EQUATIONS, SYMMETRIES, AND NUMERICAL METHOD}

We consider an incompressible flow confined in an annulus of inner radius $R_{i}$, outer radius $R_{o}$, and length $L$, driven by the constant rotation of the inner cylinder at angular speed $\Omega$, while the outer cylinder and the top and bottom end walls remain at rest. Figure 1 shows the annular geometry and includes a meridional plane with a typical streamline plot of a basic state; this meridional plane is used in subsequent figures. The system is nondimensionalized using the gap $D$ $=R_{o}-R_{i}$ as the length scale and the diffusive time across the gap $D^{2} / \nu$ as the time scale (where $\nu$ is the fluid's kine- 


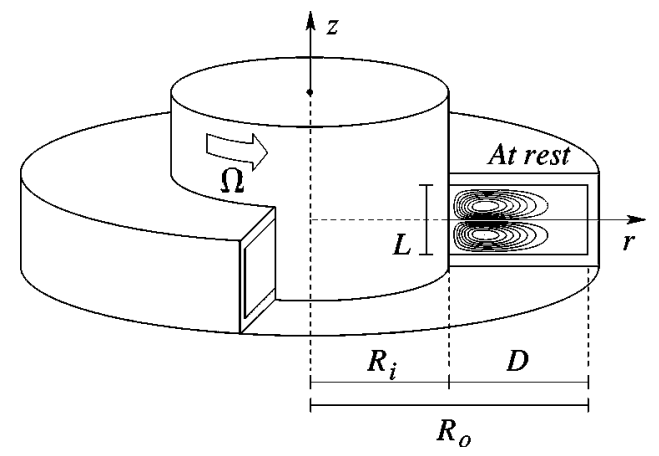

FIG. 1. Schematic of the annular flow geometry. The inset illustrates typical streamlines of a steady axisymmetric flow determined by numerical simulation, projected onto a meridional plane.

matic viscosity). The governing parameters are

$$
\begin{aligned}
\text { Reynolds number: } & \operatorname{Re}=\Omega D R_{i} / \nu, \\
\text { Aspect ratio: } & \Gamma=L / D, \\
\text { Radius ratio: } & \eta=R_{i} / R_{o} .
\end{aligned}
$$

The equations governing the flow are the Navier-Stokes equations together with initial and boundary conditions; all boundaries are no-slip. In cylindrical coordinates $(r, \theta, z)$, the nondimensional velocity vector is $\mathbf{u}=(u, v, w)$. The domain is $(r, \theta, z) \in\left[r_{i}=\eta /(1-\eta), r_{o}=1 /(1-\eta)\right] \times[0,2 \pi]$ $\times[-\Gamma / 2, \Gamma / 2]$. The discontinuities in these ideal boundary conditions at $\left(r=r_{i}, z= \pm \Gamma / 2\right)$ physically correspond to small but finite gaps between the rotating cylinder and the stationary end walls. For an accurate use of spectral techniques, a regularization of these discontinuities is implemented of the form

$$
u=w=0, v=\operatorname{Re} \exp \left[-\left(\frac{r-r_{i}}{\epsilon}\right)^{2}\right],
$$

for $r \in\left[r_{i}, r_{o}\right]$ and where $\epsilon$ is a small parameter that mimics the small gaps (we have used $\epsilon=0.005$ ). The use of $\epsilon \neq 0$ regularizes the otherwise discontinuous boundary conditions. See Refs. [16,17] for further details of the use of this regularization in spectral codes.

The three-dimensional Navier-Stokes equations are solved numerically using a Galerkin spectral scheme for spatial discretization and a second-order projection scheme for time evolution. Legendre polynomial bases are used in the radial and axial directions and a Fourier basis is used in the periodic azimuthal direction. The details of the numerical method are given in Ref. [17]. For all the computed results presented here, 48 Legendre modes in $r$ and $z, 32$ Fourier modes in $\theta$, and a time step of $2 \times 10^{-4}$ have been used.

The governing equations and boundary conditions are equivariant to rotations $R_{\alpha}$, of arbitrary angle $\alpha$, around the cylinder axis, and also to a specular reflection $K$ about the midplane $z=0$. Their action on the velocity vector $\mathbf{u}$ is

$$
R_{\alpha}(u, v, w)(r, \theta, z)=(u, v, w)(r, \theta+\alpha, z),
$$

$$
K(u, v, w)(r, \theta, z)=(u, v,-w)(r, \theta,-z)
$$

Since $R_{\alpha}$ and $K$ commute, the symmetry group of the problem is $\mathcal{G}=\mathrm{SO}(2) \times Z_{2}$. The basic state, i.e., the unique solution of the Navier-Stokes equations for small values of Re, is steady and invariant to the group $\mathcal{G}$.

Using the spectral code, it is straightforward to consider the dynamics in a number of invariant subspaces: (i) the $\mathrm{SO}(2) \times Z_{2}$-invariant subspace, where all solutions are axisymmetric and reflection symmetric about $z=0$, (ii) the $\mathrm{SO}(2)$-invariant subspace, where all solutions are axisymmetric but the midplane $(z=0)$ need not be a symmetry plane, (iii) the $Z_{2}$-invariant subspace, where the mid-plane $(z=0)$ is a symmetry plane but the solutions need not be axisymmetric, and finally (iv) the full problem where no symmetry conditions are imposed. The restriction to the axisymmetric $\mathrm{SO}(2)$-invariant subspace is accomplished by setting to zero all but the zeroth Fourier mode; the restriction to the $Z_{2}$-invariant subspace is simply accomplished by setting to zero all the odd Legendre polynomials in the $z$ basis for $u$ and $v$ and all the even Legendre polynomials in the $z$ basis for $w$. This enforces the condition

$$
u_{z}=v_{z}=w=0
$$

at the midheight plane $(z=0)$, which means that on this plane there is no flow-through (maintaining it flat) and there are no tangential stresses. The restriction to the $\mathrm{SO}(2) \times Z_{2}$-invariant subspace is accomplished by the dual restriction to both $\mathrm{SO}(2)$ and $Z_{2}$ invariance. For a recent application of this numerical technique and symmetry methods, to a problem with similar geometry, see Ref. [18].

\section{THE BASIC STATE}

The basic state is steady, axisymmetric and reflection symmetric about the half-height plane. It is driven by the constant rotation of the inner cylinder, which imparts angular momentum to the fluid adjacent to it. In the Stokes limit $(\mathrm{Re} \rightarrow 0)$, the angular momentum is distributed throughout the annular gap by diffusion, and as Re is increased, this redistribution is also accomplished by advection. For the basic state, the advection of angular momentum results in establishing a jet flow from the inner cylinder and centered on the half-height plane. A typical basic state in a regime dominated by advection of angular momentum is shown in Fig. 2, at $\operatorname{Re}=540, \eta=0.675$, and $\Gamma=0.5$. The structure of this jet is clear from the contours of the radial and azimuthal velocity components in parts (a) and (b) of the figure. The flow is virtually stagnant in the outer one-third of the annulus, and a two-cell counter-rotating meridional flow is established to balance the fluid advected by the jet. These cells return swirling fluid back towards the inner cylinder along the stationary end walls, as illustrated by the radial velocity in part (a) of the figure. The meridional flow is concentrated in the inner one-third of the annulus, as seen in the axial velocity (part c), which is directed in towards the midheight plane $z=0$ to feed the jet.

As $\mathrm{Re}$ is increased beyond about 550, in the parameter 

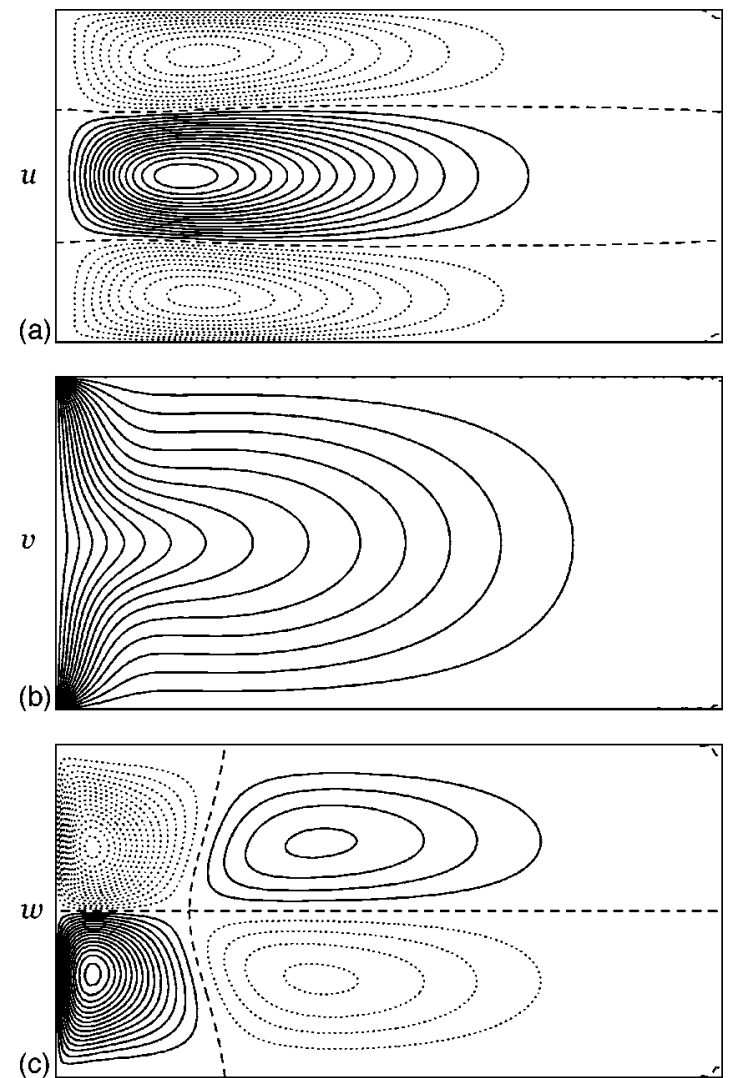

FIG. 2. Contours of velocity components $u, v$, and $w$ for the steady $\mathrm{SO}(2) \times Z_{2}$ symmetric basic state at $\mathrm{Re}=540, \eta=0.675$, and $\Gamma=0.5$. Contour levels are uniformly spaced in the ranges $u \in[-55,55], v \in[0,540]$, and $w \in[-30,30]$; the positive (negative) contours are solid (dotted) and the zero contour is dashed.

regime $\Gamma=0.5$ and $\eta \in(0.65,0.70)$, we have found that the basic state loses stability as the jet described above becomes unstable. It loses stability in two distinct ways via Hopf bifurcations.

\section{HOPF BIFURCATIONS FROM THE BASE STATE}

For a generic Hopf bifurcation, a pair of complex conjugate eigenvalues, $\pm i \omega_{0}$, cross the imaginary axis as a parameter $\mu$ is varied through $\mu=0$. According to the center manifold theorem (for infinite-dimensional systems, certain technical requirements must be satisfied in order to invoke the theorem; e.g., see Ref. [19] for details; the Navier-Stokes equations fulfill these requirements), there exists a twodimensional center manifold $\mathcal{M}_{c}$ and a normal form on it describing the dynamics of the system in a neighborhood of the bifurcation point. Using the complex amplitude of the eigenvector corresponding to the eigenvalue $i \omega_{0}$ to parametrize $\mathcal{M}_{c}$, the normal form has the form

$$
\dot{A}=i \omega_{0} A+P(A, \bar{A}, \mu),
$$

where $P$ satisfies

$$
P\left(e^{-i \omega t} A, e^{i \omega t} \bar{A}, \mu\right)=e^{-i \omega t} P(A, \bar{A}, \mu),
$$

for all $t$, from which the standard normal form for the Hopf bifurcation (to third order) is easily derived:

$$
\dot{A}=A\left(i \omega_{0}+\mu-a|A|^{2}\right) \text {. }
$$

The symmetries of the system impose additional conditions on the normal form. If the governing equations are equivariant with respect to $\mathcal{G}$, then so is the normal form. The action of $\mathcal{G}$ on the amplitude $A$ is (see Ref. [12] for details)

$$
R_{\alpha} A=e^{i m \alpha} A, \quad K A=s A,
$$

where $m$ is an integer and $s= \pm 1$. When $m=0$ the eigenvector is $\mathrm{SO}(2)$ invariant, i.e., axisymmetric; when $s=+1$, the eigenvector is $Z_{2}$ invariant, i.e., $u$, and $v$ are even functions of $z$, and $w$ is odd.

The additional conditions imposed by the symmetry group $\mathcal{G}$ on $P$ are

$$
\begin{gathered}
P\left(e^{i m \alpha} A, e^{-i m \alpha} \bar{A}, \mu\right)=e^{i m \alpha} P(A, \bar{A}, \mu), \\
P(s A, s \bar{A}, \mu)=s P(A, \bar{A}, \mu) .
\end{gathered}
$$

However, since these conditions can be obtained from Eq. (6), i.e., letting $t=-m \alpha / \omega_{0}$ gives Eq. (9), and letting $t$ $=\pi / \omega$ gives Eq. (10) (when $s=-1)$, there are no additional restrictions on the normal form due to the symmetry group $\mathrm{SO}(2) \times Z_{2}$. The action of $\mathcal{G}$ on the periodic bifurcated solution $\gamma$ is the following: if $m=0, \mathrm{SO}(2)$ leaves every point of $\gamma$ invariant. If $m \neq 0$, the action of $R_{\alpha}$ on $\gamma$ is equivalent to a time translation $t \rightarrow t+m \alpha / \omega_{0}$, and $\gamma$ is a rotating wave with precession frequency $\omega_{p}=\omega_{0} / m$. If $s=1, Z_{2}$ leaves every point of $\gamma$ invariant. If $s=-1$, the action of the $z$-flip $K$ is equivalent to a time translation of $\pi / \omega_{0}$, which is half the period of $\gamma$.

The bifurcated limit cycle $\gamma$, as a set, is $\mathcal{G}$-invariant, but the individual points on $\gamma$ (the solution at a given time) are only invariant to a subgroup $\Delta$ of $\mathcal{G}$, called the group of spatial symmetries of the bifurcated periodic solution. That is, taking a point on $\gamma$ at a particular time and applying the symmetry group $\mathcal{G}$ generally does not leave the point invariant, but results in another $\mathcal{G}$-conjugate point on $\gamma$. If instead the subgroup $\Delta$ is applied to the point, it remains invariant. The remaining elements of $\mathcal{G}$ are called spatiotemporal symmetries of $\gamma$, and their action is equivalent to a specified time translation along the orbit. The quotient group $\mathcal{G} / \Delta$ is always $\mathrm{SO}(2)$ or $Z_{m}$ (see Ref. [20] for a much more general and complete discussion of the symmetries of periodic solutions and their possible bifurcations). Here $Z_{m}$, also called $C_{m}$, is the cyclic group of $m$ elements.

There are four different possibilities for the symmetries of the bifurcated orbit $\gamma$ :

$\begin{array}{lllll} & m & s & \Delta & \mathcal{G} / \Delta \\ \text { I } & 0 & +1 & \mathrm{SO}(2) \times Z_{2} & \mathbf{1} \\ \text { II } & 0 & -1 & \mathrm{SO}(2) & Z_{2} \\ \text { III } & \neq 0 & +1 & Z_{m} \times Z_{2} & \mathrm{SO}(2) \\ \text { IV } & \neq 0 & -1 & Z_{m} & \mathrm{SO}(2)\end{array}$


where $\mathbf{1}$ is the trivial group with a single element (the identity), $Z_{m}$ is the discrete group of rotations generated by $R_{2 \pi / m}$, and $Z_{2 m}$ is generated by $K R_{\pi / m}$; the notation $Z_{m}\left(R_{2 \pi / m}\right)$ and $Z_{2 m}\left(K R_{\pi / m}\right)$ is often used to indicate simultaneously the group and the corresponding generator(s).

In the Taylor-Couette problem considered here, we have found Hopf bifurcations from the basic state of types II and IV (with $m=1$ ). They both break the $Z_{2}$ generated by $K$, the flip $z \rightarrow-z$. The physical mechanism responsible is the instability of the compound jet emanating from the inner cylinder at the midplane. The velocity field here has jetlike structure in both the radial and the azimuthal direction. When the radial component of the jet is unstable, an axisymmetric limit cycle results, and when the azimuthal component is unstable, a rotating wave with azimuthal wave number $m$ $=1$ results.

The axisymmetric limit cycle $\mathrm{LC}_{0}$, is the type-II solution and it is pointwise $\mathrm{SO}(2)$ invariant to arbitrary rotations about the axis. It breaks the pointwise $Z_{2}$ symmetry, but retains a spatiotemporal symmetry consisting of $K$ together with a half-period time translation; this symmetry generates the spatiotemporal $Z_{2}$ symmetry. The rotating wave, $\mathrm{RW}_{1}$ is a type-IV solution which breaks both the rotational and $K$ symmetries, but retains a pointwise symmetry, the product of the axial flip $K$ with a half-period rotation $K R_{\pi}$; this symmetry generates the group of spatial symmetries, $\Delta=Z_{2}$. The spatiotemporal symmetries of $\mathrm{RW}_{1}$ are arbitrary rotations $R_{\alpha}$ combined with the corresponding time translations of $\alpha / \omega$. This corresponds to the rotating wave structure, a rigid spatial pattern rotating with angular velocity $\omega . \mathrm{RW}_{1}$ also has the spatiotemporal symmetry $K$ together with a half-period time translation, as does $\mathrm{LC}_{0}$.

Figures 3 and 4 show contours of the axial velocity $w$ and its perturbation $w_{p}$ (i.e., the axial velocity minus the axial velocity of the basic state), over one period, in time or azimuth, of $\mathrm{LC}_{0}$ and $\mathrm{RW}_{1}$, respectively. The two solutions shown are at the same point in parameter space $(\operatorname{Re}=571$, $\eta=0.70, \Gamma=0.5$ ), which is in a wedge region delineated by Neimark-Sacker bifurcations in which both periodic states are stable. The Neimark-Sacker bifurcations originate at a codimension-two double Hopf point. The period of $\mathrm{LC}_{0}$ shown in the figure is $T \approx 0.0644$ and this period varies little with parameters (about 2\%), over the region of parameter space explored here. The figure illustrates the symmetries of the two periodic states. They also show that the perturbation has small magnitude at outer radii, propagates radially inwards with time for $\mathrm{LC}_{0}$ and with $\theta$ for $\mathrm{RW}_{1}$, and intensifies substantially at smaller radii. Although the perturbation, which destroys the pointwise $Z_{2}$ reflection symmetry of the solution, is small at large radii, the deflection of the zero axial velocity contour off the $z=0$ plane is greatest at large radii as the axial velocity is small there, and so the relative perturbation is large.

The two Hopf bifurcations are supercritical. This is evident from the variation of the squared amplitudes of the periodic solutions with parameters. As measures of the squared amplitudes, we use the modal energies $t$ $w$ $w_{p}$

$T / 6$

$2 T / 6$

$3 T / 6$

$4 T / 6$

$5 T / 6$
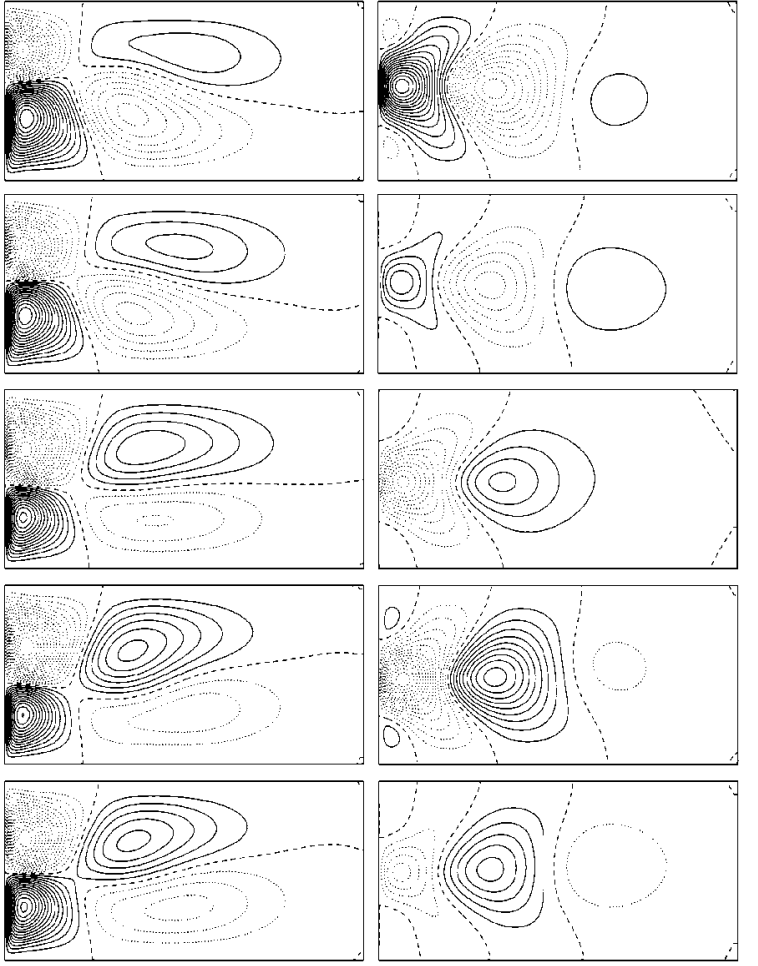

$T$
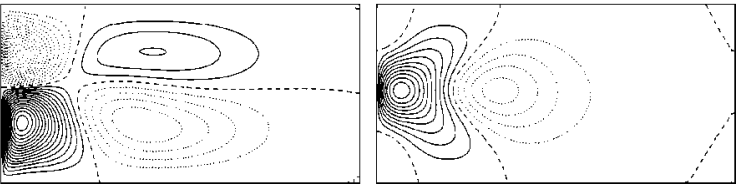

FIG. 3. Contours of (left column) axial velocity $w$ and (right column) its perturbation (i.e., $w p=w-w_{0}$ ), over six phases of the period $T \approx 0.0644$ for the axisymmetric limit cycle $\mathrm{LC}_{0}$ at $\mathrm{Re}$ $=571$ and $\eta=0.700$. Contour levels are uniformly spaced in the ranges $[-32,32]$ for $w$ and $[-16,16]$ for $w_{p}$; the positive (negative) contours are solid (dotted) and the zero contour is dashed.

$$
E_{m}=\int_{0}^{2 \pi} \int_{-\Gamma / 2}^{\Gamma / 2} \int_{\eta /(1-\eta)}^{1 /(1-\eta)} \mathbf{u}_{m} \cdot \mathbf{u}_{m}^{*} r d r d z d \theta
$$

where $\mathbf{u}_{m}$ is the $m$ th Fourier mode of the velocity field.

For the axisymmetric $\mathrm{LC}_{0}$, only $E_{0}$ is nonzero and it oscillates with a frequency $\omega$ that varies only to second order with parameters from the Hopf frequency. We refer to the amplitude of the oscillation in $E_{0}$ as $A_{\mathrm{LC}}^{2}$. In Fig. 5, $A_{\mathrm{LC}}^{2}$ for $\Gamma=0.5$ and $\eta=0.650,0.655,0.660,0.667,0.670,0.675$, $0.680,0.685,0.690,0.695$, and 0.700 is plotted as a function of Re, showing that this squared amplitude grows linearly with Re from zero at the Hopf bifurcation, $H_{\mathrm{LC}}$. For $\eta$ $>0.675, \mathrm{LC}_{0}$ is unstable to nonaxisymmetric disturbances at onset, but we can still compute this unstable state by restricting the computations to an axisymmetric subspace. The unstable $\mathrm{LC}_{0}$ becomes stable at a Neimark-Sacker bifurcation $N_{\text {LC}}$.

For $\mathrm{RW}_{1}$, there are nonzero modal energies $E_{0}$ and $E_{1}$ $\left(E_{n} \neq 0\right.$ for $n>1$, but these are simply harmonics of $\left.E_{1}\right)$. However, unlike for the case with $\mathrm{LC}_{0}, E_{0}$ and $E_{1}$ for $\mathrm{RW}_{1}$ 


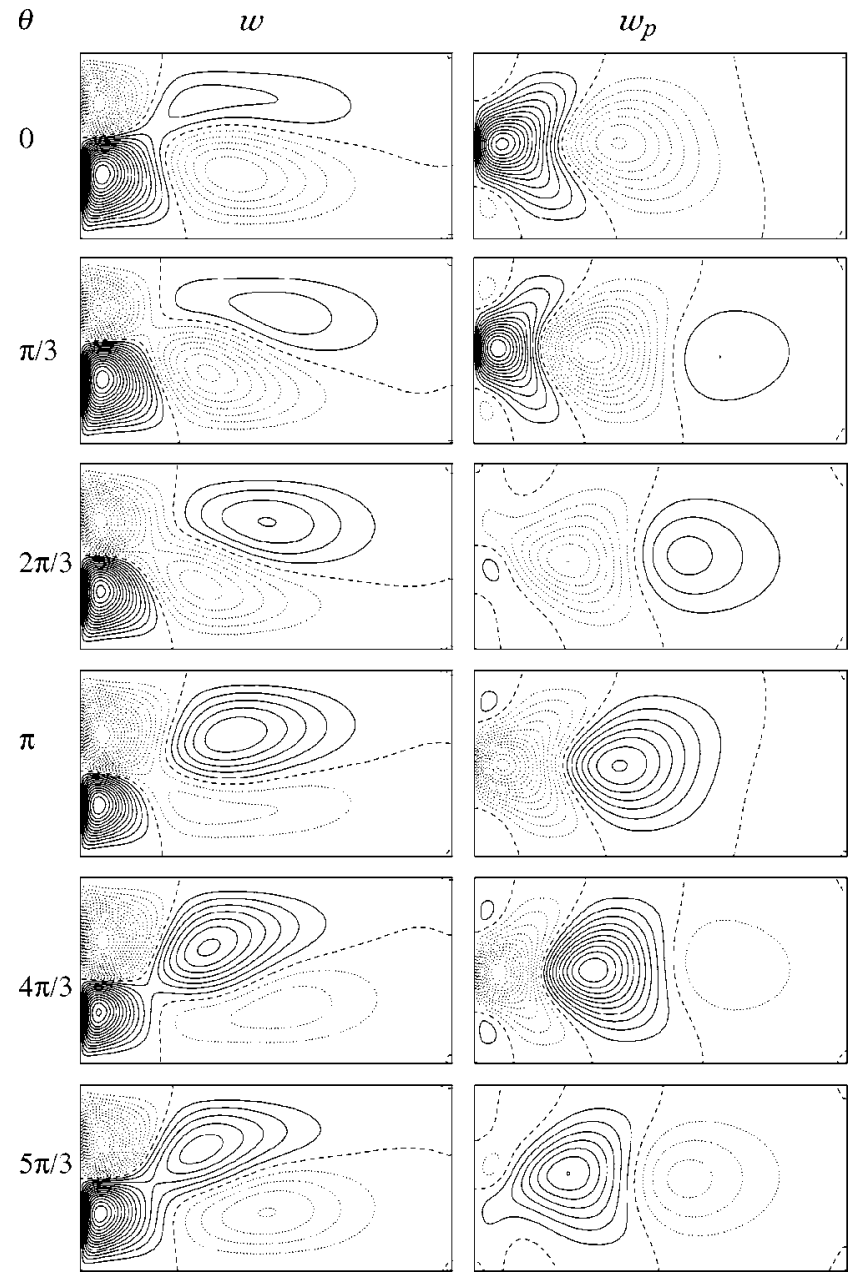

FIG. 4. Contours of (left column) axial velocity $w$ and (right column) its perturbation (i.e., $w p=w-w_{0}$ ), in six meridional planes (at angle $\theta$ ), for the $\mathrm{RW}_{1}$ state at $\mathrm{Re}=571$ and $\eta=0.700$. Contours levels are uniformly spaced in the ranges [-32,32] for $w$ and $[-16,16]$ for $w_{p}$; the positive (negative) contours are solid (dotted) and the zero contour is dashed.

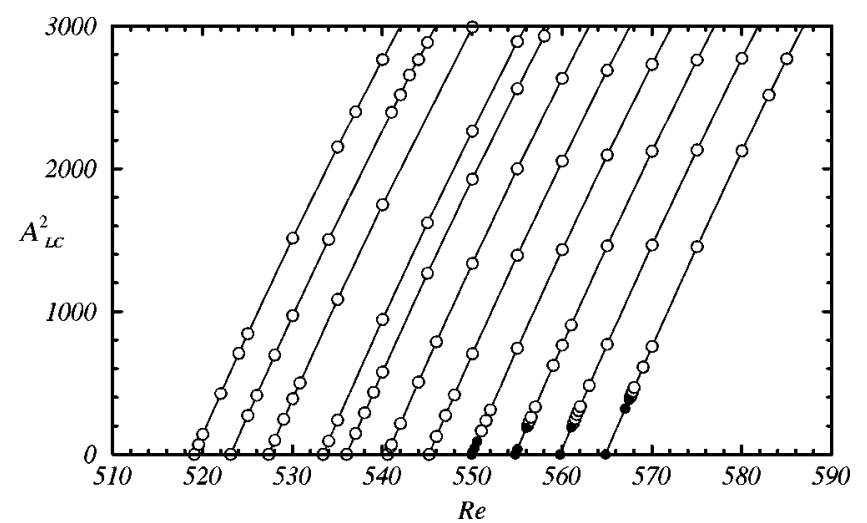

FIG. 5. Variation of $A_{\mathrm{LC}}^{2}$, a measure of the squared amplitude of the axisymmetric limit cycle $\mathrm{LC}_{0}$, with $\mathrm{Re}$ for $\Gamma=0.5$ and $\eta$ $=0.650,0.655,0.660,0.667,0.670,0.675,0.680,0.685,0.690$, $0.695,0.700$; the small $\eta$ curves start at small Re. Open (filled) circles denote stable (unstable) states.

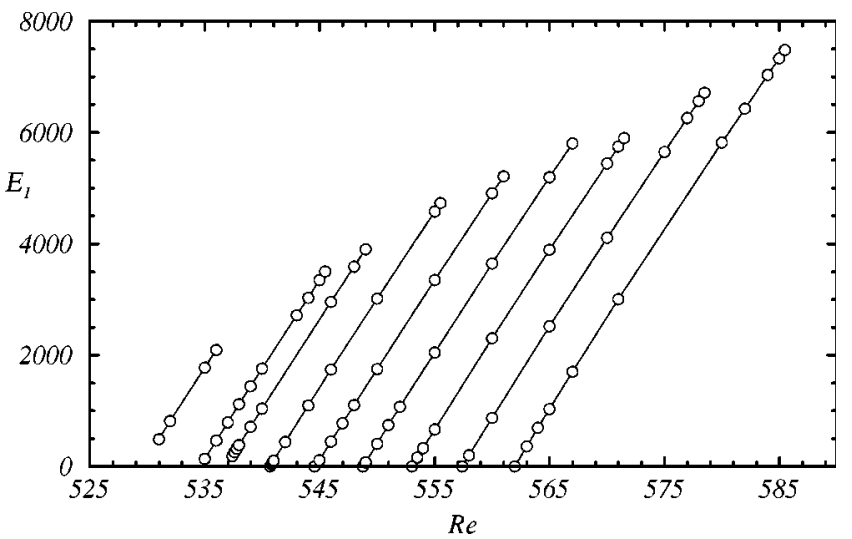

FIG. 6. Variation of $E_{1}$, a measure of the squared amplitude of the $m=1$ rotating wave $\mathrm{RW}_{1}$, with $\mathrm{Re}$ for $\Gamma=0.5$ and $\eta=0.660$, $0.667,0.670,0.675,0.680,0.685,0.690,0.695,0.700$; the small $\eta$ curves start at small Re.

are both constant in time. As a direct measure of the squared amplitude of $\mathrm{RW}_{1}$, we use $E_{1}$. In Fig. 6, $E_{1}$ for $\mathrm{RW}_{1}$ at $\Gamma$ $=0.5$ and $\eta=0.660,0.667,0.670,0.675,0.680,0.685$, $0.690,0.695$, and 0.700 are plotted as functions of Re, also showing linear growth with Re from onset, i.e., we again have a supercritical Hopf bifurcation $H_{\mathrm{RW}}$. For $\eta<0.675$, $\mathrm{RW}_{1}$ bifurcates supercritically from a basic state that is already unstable to $\mathrm{LC}_{0}$. Unlike with the case of unstable $\mathrm{LC}_{0}$, we do not have a subspace in which the unstable $\mathrm{RW}_{1}$ is stable, and so have difficulty in following this unstable timeperiodic state using the time-evolution code. The unstable $\mathrm{RW}_{1}$ becomes stable at a Neimark-Sacker bifurcation $N_{\mathrm{RW}}$.

The two curves of Hopf bifurcations, $H_{\mathrm{LC}}$ and $H_{\mathrm{RW}}$, intersect at a codimension-two double Hopf bifurcation at $\left(\mathrm{Re}_{\mathrm{dH}} \approx 541, \eta_{\mathrm{dH}} \approx 0.676\right)$ for $\Gamma=0.5$.

\section{DOUBLE HOPF BIFURCATION FROM THE BASIC STATE}

The two periodic solutions $\mathrm{LC}_{0}$ and $\mathrm{RW}_{1}$ bifurcate simultaneously at a codimension-two double Hopf bifurcation point. The double Hopf bifurcation with $\mathrm{SO}(2)$ symmetry has been described in Ref. [21], and the one considered here is of a nonresonant simple type. The corresponding normal form, including the effect of $\mathrm{SO}(2)$, is

$$
\begin{aligned}
& \dot{A}=i \omega_{0} A+\widetilde{P}(A, B, \bar{A}, \bar{B}, \mu)=A\left[i \omega_{0}+P\left(|A|^{2},|B|^{2}, \mu\right)\right], \\
& \dot{B}=i \omega_{1} B+\widetilde{Q}(A, B, \bar{A}, \bar{B}, \mu)=B\left[i \omega_{1}+Q\left(|A|^{2},|B|^{2}, \mu\right)\right],
\end{aligned}
$$

where $A$ and $B$ are the complex amplitudes of the eigenfunctions corresponding to $\mathrm{LC}_{0}$ and $\mathrm{RW}_{1}$, and $\mu$ are parameters. The additional symmetry $Z_{2}(K)$ imposes further conditions on $\widetilde{P}$ and $\widetilde{Q}$ :

$$
\begin{aligned}
\widetilde{P}(-A,-B,-\bar{A},-\bar{B}, \mu) & =-\widetilde{P}(A, B, \bar{A}, \bar{B}, \mu), \\
\widetilde{Q}(-A,-B,-\bar{A},-\bar{B}, \mu) & =-\widetilde{Q}(A, B, \bar{A}, \bar{B}, \mu) .
\end{aligned}
$$




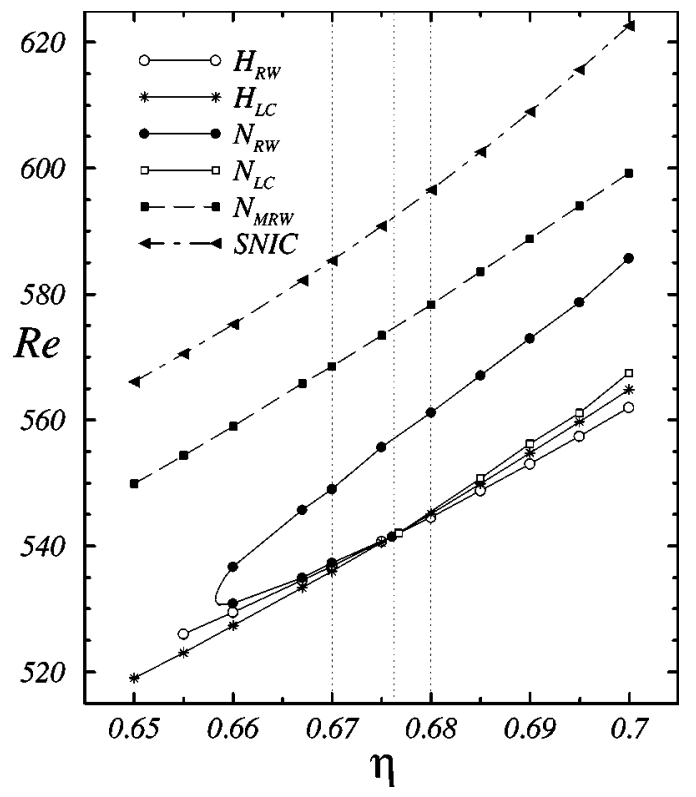

FIG. 7. Stability diagram in $(\eta, \mathrm{Re})$ parameter space (with $\Gamma$ $=0.5$, showing the various bifurcation curves. $H_{\mathrm{LC}}$ and $H_{\mathrm{RW}}$ are Hopf bifurcations to $\mathrm{LC}_{0}$ and $\mathrm{RW}_{1}$, respectively. $N_{\mathrm{LC}}, N_{\mathrm{RW}}$, and $N_{\text {MRW }}$ are Neimark-Sacker bifurcations, and SNIC is the saddlenode-on-invariant-circle bifurcation leading to the VLF.

These conditions are satisfied by $P$ and $Q$, and the symmetry group $\mathcal{G}=\mathrm{SO}(2) \times Z_{2}$ does not modify the normal form from that of the generic double Hopf bifurcation; the symmetries only manifests themselves in the symmetry properties of the bifurcated solutions.

The analysis of the double Hopf normal form, Eqs. (13) and (14), follows exactly the same lines as in Ref. [21], and results in a wedge-shaped region emerging from the double Hopf point where both bifurcated solutions $\mathrm{LC}_{0}$ and $\mathrm{RW}_{1}$ coexist and are simultaneously stable. This region is separated from the regions where only one of the bifurcated solutions is stable by Neimark-Sacker curves, where one of the two stable coexisting solutions become unstable. At the Neimark-Sacker curves $N_{\mathrm{LC}}$ and $N_{\mathrm{RW}}$, the unstable $\mathrm{LC}_{0}$ and the unstable $\mathrm{RW}_{1}$ become stable, respectively, as an unstable mixed mode (a modulated rotating wave) is spawned in the wedge region. Since this mixed mode is unstable, it cannot be observed experimentally, or computed with a timeevolution code. In our problem, in the neighborhood of the double Hopf point, the two Hopf curves and two NeimarkSacker curves vary linearly with parameters, as indicated in the stability diagram in Fig. 7, for $\Gamma=0.5$. In the neighborhood of the double Hopf bifurcation, the two Hopf curves $H_{\mathrm{LC}}$ and $H_{\mathrm{RW}}$ and the two Neimark-Sacker curves $N_{\mathrm{LC}}$ and $N_{\mathrm{RW}}$ are close to being tangent. As we follow $N_{\mathrm{RW}}$ away from the double Hopf point, it curves around so that there is no stable $\mathrm{RW}_{1}$ for $\eta<0.658$; also at fixed $\eta>0.658$, as Re is increased $\mathrm{RW}_{1}$ loses stability as the unstable mixed mode is re-absorbed at $N_{\mathrm{RW}}$. For higher Re, the only stable state is $\mathrm{LC}_{0}$, until it undergoes another Neimark-Sacker bifurcation $N_{\text {MRW }}$, which is not related to the local dynamics associated with the double Hopf bifurcation.

\section{NEIMARK-SACKER BIFURCATION TO A MODULATED ROTATING WAVE}

Both $\mathrm{LC}_{0}$ and $\mathrm{RW}_{1}$ have a space-time $Z_{2}$ symmetry, the spatial reflection $K$ followed by a half-period time translation. The Poincaré map $\mathbf{P}$ is the square of the half-period flip map:

$$
\begin{aligned}
\mathbf{H}: \quad E & \mapsto E \\
x & \mapsto \mathbf{H} x=K \phi(T / 2 ; x),
\end{aligned}
$$

where $E$ is a local Poincare section of $\mathrm{RW}_{1}, T$ is approximately the precession period of $\mathrm{RW}_{1}$ ( $\mathbf{H}$ is the first hit map of $E$ ), and $\phi$ is the flow of the system. The theory of bifurcations from limit cycles with $Z_{2}$ symmetry can be found in Ref. [22], and it relies mainly on the properties of the halfperiod flip map $\mathbf{H}$. In the present case, both limit cycles are of $S$ type in Kuznetsov's notation (which means that $K$ is not a space symmetry, but a space-time symmetry for the cycles). Due to the presence of $\mathbf{H}$, period-doubling bifurcations are inhibited [23], and for bifurcations with eigenvalues of multiplicity 1 , there remain three possible cases:

(1) The eigenvector is invariant to $\mathbf{H}$, and the critical Floquet multiplier of $\mathbf{P}, \lambda_{P}$, and the critical Floquet multiplier of $\mathbf{H}, \lambda_{H}$, are both 1 . This is a saddle-node bifurcation of periodic solutions, and the bifurcated solutions retain the space-time symmetry $\mathbf{H}$.

(2) The eigenvector is not invariant to $\mathbf{H}, \lambda_{H}=-1$, and $\lambda_{P}=\lambda_{H}^{2}=1$. This is a pitchfork bifurcation of periodic solutions for the map $\mathbf{H}$, and the bifurcated solutions break the space-time symmetry $\mathbf{H}$; the two bifurcated limit cycles are symmetrically related by $K$. These still correspond to a single $T$-periodic state for the map $\mathbf{P}$, which is not of $S$-type.

(3) There are a pair of complex conjugate eigenvectors, and a complex conjugate pair of eigenvalues for both maps $\mathbf{H}$ and $\mathbf{P}$. This is a Neimark-Sacker bifurcation, and the bifurcated two-torus is $K$ invariant, although both $K$ and $\mathbf{H}$ transform a given solution on the two-torus into a different solution on the same two-torus.

The bifurcations of $\mathrm{LC}_{0}$ and $\mathrm{RW}_{1}$ in our system have been observed to be of Neimark-Sacker type. Close to the double Hopf bifurcation point, where the two solutions coexist and are stable, an unstable modulated rotating wave also exists, as previously mentioned. Far from the double Hopf bifurcation point, $\mathrm{LC}_{0}$ loses stability in a NeimarkSacker bifurcation, $N_{\mathrm{MRW}}$, and a stable modulated rotating wave (MRW) emerges. This Neimark-Sacker curve is indicated in Fig. 7.

The Neimark-Sacker bifurcation $N_{\text {MRW }}$ is supercritical. Figure 8 shows the linear growth of $\bar{E}_{1}$ with Re for $\Gamma=0.5$ and $\eta=0.650,0.655,0.660,0.667,0.670,0.675,0.680$, $0.685,0.690,0.695,0.700$; where $\bar{E}_{1}$ is the time average of $E_{1}$ for MRW. Over the range of Re that MRW exists, it is the only stable state. By increasing Re in small increments, MRW is followed until beyond a critical value (for a given value of $\eta$ ), the solution switches from being a two-torus to a three-torus solution, consisting of two periods that are approximately the same as those of MRW, plus a third much longer period. We surmise that the stable MRW which had 


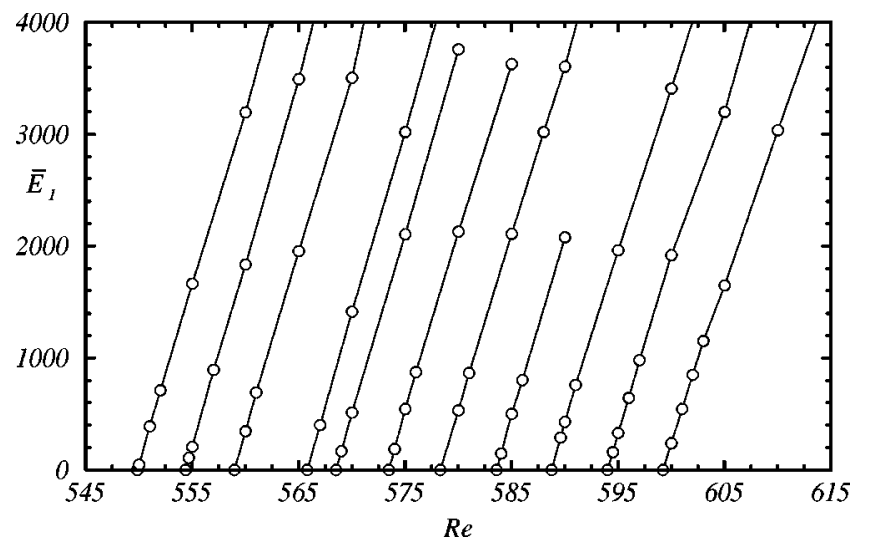

FIG. 8. Variation of $\bar{E}_{1}$, the mean kinetic energy in the azimuthal mode $m=1$ of MRW, with $\operatorname{Re}$ at $\Gamma=0.5$ and $\eta=0.650$, $0.655,0.660,0.667,0.670,0.675,0.680,0.685,0.690,0.695,0.700$; the curves at lower Re correspond to lower $\eta$.

bifurcated from the stable $\mathrm{LC}_{0}$ at $N_{\mathrm{MRW}}$, collides with an unstable (saddle) MRW which bifurcated from the unstable $\mathrm{RW}_{1}$ at another $N_{\mathrm{MRW}}$, following the destabilization of $\mathrm{RW}_{1}$ at $N_{\mathrm{RW}}$. This saddle-node bifurcation between the stable and saddle MRW results in a saddle-node-infinite-period bifurcation, which spawns the three-torus very-low-frequency state VLF. Schematics of the whole bifurcation sequences, with increasing $\operatorname{Re}$ for $\eta$ at either side and at the double Hopf bifurcation, are shown in Fig. 9.

\section{SADDLE-NODE-INFINITE-PERIOD BIFURCATION}

SNIC stands for saddle-node on an invariant cycle, also known as saddle-node-infinite-period bifurcation. In this bifurcation, studied by Andronov, Leontovich, and Shil'nikov (see Ref. [22]), a saddle-node bifurcation takes place on an invariant cycle, as illustrated in Fig. 10. Before the bifurcation, the invariant circle is a periodic solution. Its period grows to infinity as the bifurcation point is approached, obeying an inverse square-root law with the distance to the bifurcation in parameter space $[22,24]$. After the bifurcation, we have a saddle and a node bifurcated solutions, connected via two heteroclinic curves, which form the invariant circle that continues existing after the bifurcation. SNIC bifurcations usually occur in conjunction with Neimark-Sacker bifurcations and form the boundaries of the Arnold tongues that are present in a neighborhood of a Neimark-Sacker bifurcation. However, they can also exist without associated resonant dynamics, as is the case in the problem presented here.

In our problem, the bifurcation takes place as $\mathrm{Re}$ is decreased; i.e., we have a stable (node) MRW that collides with an unstable (saddle) MRW in a saddle-node bifurcation (we do not directly compute the unstable MRW). After the collision, but in its neighborhood, what is left at higher Re is a very long period solution that we have termed the VLF. In the standard SNIC bifurcation, the saddle and node that collide are fixed points, and the limit cycle is a periodic solution. In our case, the solutions that collide are two-tori, modulated rotating waves, and the emerging VLF state is a
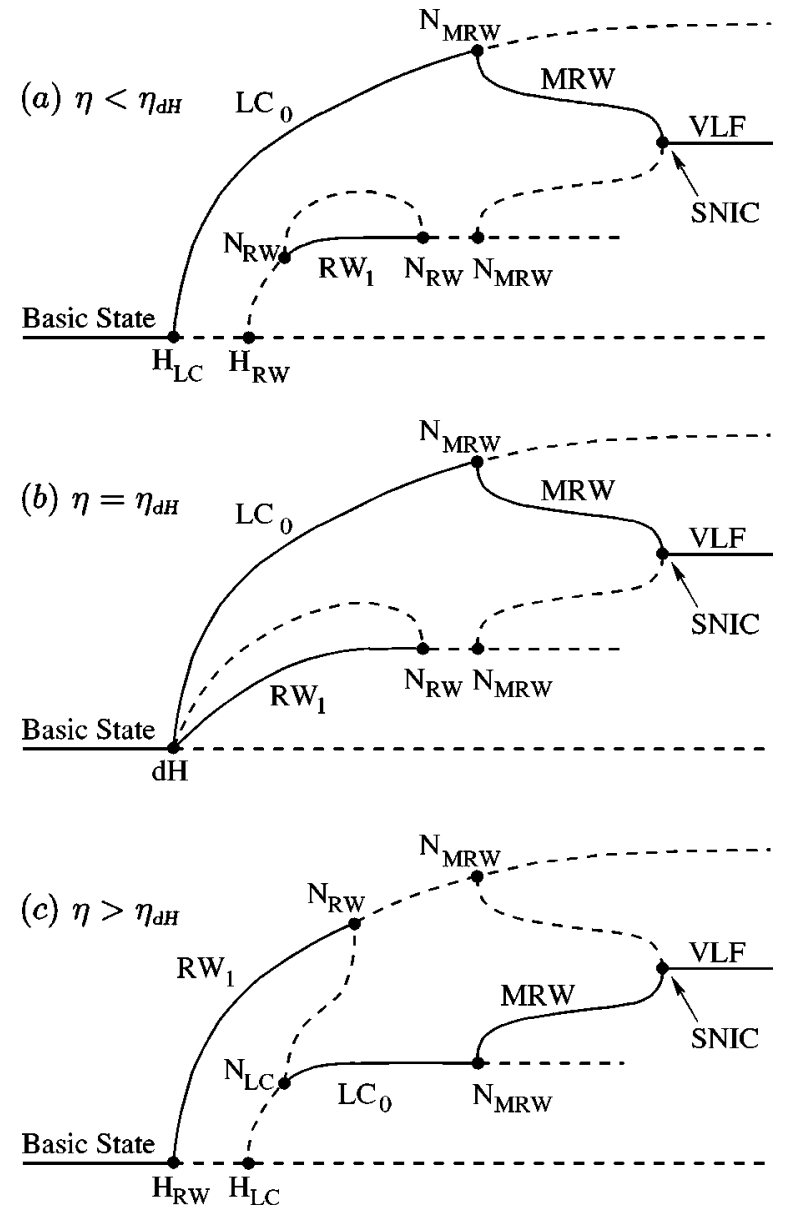

FIG. 9. Schematic bifurcation diagrams, using Re as the bifurcation parameter, fixing $\Gamma=0.5$ and for (a) $\eta<\eta_{\mathrm{dH}}$, (b) $\eta=\eta_{\mathrm{dH}}$, and (c) $\eta=\eta_{\mathrm{dH}} . H_{\mathrm{LC}}$ and $H_{\mathrm{RW}}$ are Hopf bifurcations to $\mathrm{LC}_{0}$ and $\mathrm{RW}_{1}$, respectively, and $d H$ is the codimension-two bifurcation where both $H_{\mathrm{LC}}$ and $H_{\mathrm{RW}}$ occur simultaneously. $N_{\mathrm{LC}}, N_{\mathrm{RW}}$, and $N_{M R W}$ are Neimark-Sacker bifurcations, and SNIC is the saddlenode-on-invariant-circle bifurcation leading to the VLF state. Stable (unstable) solution branches are solid (dashed) curves.

stable and robust three-torus, that exists in an extensive range of parameter space. It has two frequencies which correspond to the axisymmetric oscillation of MRW and the precession of the $m=1$ component of MRW. The third new period $T_{\mathrm{VLF}}$ becomes unbounded at the SNIC bifurcation. Figure 11 shows the variation of $T_{\mathrm{VLF}}$ with $\operatorname{Re}$ for $\Gamma=0.5$ and $\eta=0.650,0.655,0.660,0.667,0.670,0.675,0.680$, $0.685,0.690,0.695,0.700$. The circles are the computed periods and the lines are fits of the form $T_{\mathrm{VLF}}=a_{0}$ $+a_{1} / \sqrt{\mathrm{Re}-\mathrm{Re}_{\mathrm{VLF}}}$. Sample time series of $E_{0}$ (the modal energy in the axisymmetric component of the flow), for VLF are shown in Fig. 12. As Re approaches the SNIC bifurcation, the time series approaches that corresponding to MRW, except for the rapid periodic excursions.

\section{DISCUSSION AND CONCLUSION}

The short aspect ratio of the annulus investigated here results in a basic state with a single outward jet at the mid- 
(a) $R e=582.4$

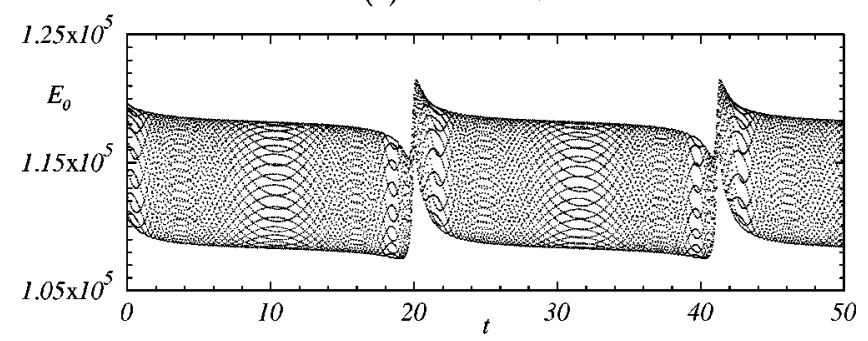

(b) $R e=584.0$

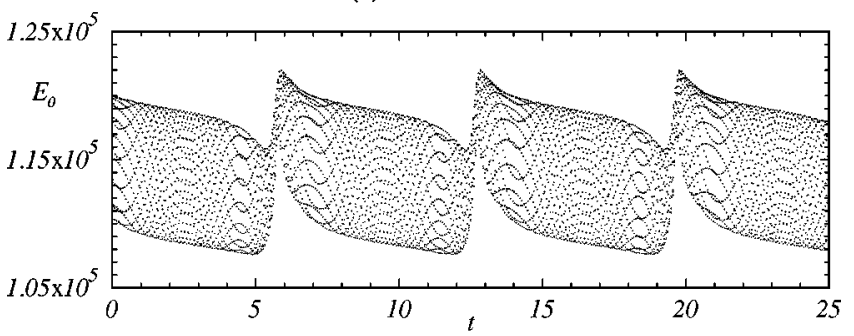

(c) $R e=588.0$

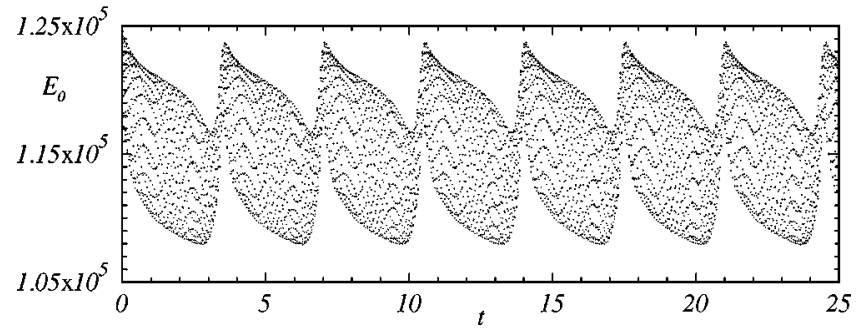

FIG. 12. Time series of $E_{0}$ for the VLF state at $\Gamma=0.5, \eta$ $=0.667$, and Re as indicated (a dot is drawn every 20th time step in the computation, where $\delta t=2 \times 10^{-4}$ ).

tions at higher Reynolds numbers. It is stable (unstable) when it bifurcates from a stable (unstable) periodic state. These stable and unstable modulated rotating waves are heteroclinically connected, and with increasing Reynolds number, collide and vanish in a saddle-node bifurcation. Following the collision, all that remains is an invariant manifold consisting of the previous heteroclinic connections. This invariant manifold is a stable three-torus, with two frequencies corresponding to those of the modulated rotating waves, and a third very low frequency that vanishes at the SNIC. This very-low-frequency state has been previously observed in experiments [6,25], but here we present the first computed example of such a state, together with a comprehensive bifurcation sequence leading to its onset.

The very-low-frequency states have been observed experimentally in a number of Taylor-Couette flows where end wall effects are important, i.e., in short aspect ratio systems, although they have also been observed in experiments with aspect ratios of order 10. Also, the appearance of the VLF state has not only been associated with a saddle-nodeinfinite-period bifurcation, but has also been associated with cycle-saddle homoclinic collisions in the same experiments but in different parameter regimes $[8,25]$. The two global bifurcations are distinguished by the scaling law describing how the associated period $T_{\mathrm{VLF}}$ becomes unbounded. With the saddle-node-infinite-period bifurcation, 
$T_{\mathrm{VLF}} \sim 1 / \sqrt{\left|\mathrm{Re}-\mathrm{Re}_{\mathrm{VLF}}\right|} ;$ and for the homoclinic collision $T_{\mathrm{VLF}} \sim 1 / \mathrm{ln}\left|\operatorname{Re}-\mathrm{Re}_{\mathrm{VLF}}\right|$; which one is observed seems to depend on the path through parameter space taken. If the basic state first loses stability via a Hopf bifurcation where the resulting periodic state is setwise $Z_{2}$ invariant, then it appears that the onset of a VLF state occurs via the saddlenode-infinite-period bifurcation. If on the other hand, the basic state loses stability via a steady pitchfork bifurcation, producing two conjugate steady states that subsequently become unstable via Hopf bifurcations that result in a pair of limit cycles that are not $Z_{2}$ invariant, but are conjugate under a $Z_{2}$ reflection, then these may undergo a gluing bifurcation where the two cycles simultaneously collide homoclinically with the unstable (saddle) basic state. This gluing bifurcation produces a new $Z_{2}$-invariant cycle with an associated very low frequency that obeys a $\ln \left|\operatorname{Re}-\mathrm{Re}_{\mathrm{VLF}}\right|$ law. Such a gluing bifurcation in a short annulus Taylor-Couette experiment has recently been reported [10]. All this suggests that the two global bifurcations may be organized by a Takens-Bogdanov bifurcation at which a Hopf and a pitchfork bifurcation coincide. Recently, Rucklidge [26] has studied the dynamics associated with the normal form of a Takens-Bogdanov bifurcation with $D_{4}$ symmetry and has identified a scenario that spawns both types of global bifurcations. Although the symmetries of his problem differ from those of ours, there are sufficient features in common that suggest that the VLF states may well be organized by such a bifurcation. Investigations into this conjecture are currently underway.

\section{ACKNOWLEDGMENTS}

This work was supported by the NSF through Grant No. CTS-9908599 (USA) and MCyT Grant No. BFM2001-2350 (Spain).
[1] D. Coles, J. Fluid Mech. 21, 385 (1965).

[2] H.L. Swinney and J.P. Gollub, Hydrodynamic Instabilities and the Transition to Turbulence (Spriger-Verlag, Berlin, 1981).

[3] P. Chossat and G. Iooss, The Couette-Taylor Problem (Springer, New York, 1994).

[4] T.B. Benjamin, Proc. R. Soc. London, Ser. A 359, 1 (1978).

[5] T.B. Benjamin and T. Mullin, Proc. R. Soc. London, Ser. A 377, 221 (1981).

[6] G. Pfister, H. Schmidt, K.A. Cliffe, and T. Mullin, J. Fluid Mech. 191, 1 (1988).

[7] T. Mullin, S.J. Tavener, and K.A. Cliffe, Europhys. Lett. 8, 251 (1989).

[8] T.J. Price and T. Mullin, Physica D 48, 29 (1991).

[9] J. von Stamm, U. Gerdts, T. Buzug, and G. Pfister, Phys. Rev. E 54, 4938 (1996).

[10] J. Abshagen, G. Pfister, and T. Mullin, Phys. Rev. Lett. 87, 224501 (2001).

[11] M. Golubitsky, I. Stewart, and D.G. Schaeffer, Singularities and Groups in Bifurcation Theory (Springer, New York, 1988), Vol. II.

[12] G. Iooss and M. Adelmeyer, Topics in Bifurcation Theory and Applications, 2nd ed. (World Scientific, Singapore, 1998).

[13] P. Chossat and R. Lauterbach, Methods in Equivariant Bifurcations and Dynamical Systems (World Scientific, Singapore, 2000).
[14] T. Mullin, Y. Toya, and S.J. Tavener, Phys. Fluids 14, 2778 (2002).

[15] H. Furukawa, T. Watanabe, Y. Toya, and I. Nakamura, Phys. Rev. E 65, 036306 (2002).

[16] J.M. Lopez and J. Shen, J. Comput. Phys. 139, 308 (1998).

[17] J.M. Lopez, F. Marques, and J. Shen, J. Comput. Phys. 176, 384 (2002).

[18] F. Marques, A.Y. Gelfgat, and J.M. Lopez, Phys. Rev. E 68, 016310 (2003).

[19] J. E. Marsden and M. McCracken, The Hopf Bifurcation and Its Applications, Applied Mathematical Sciences (Springer, New York, 1976), Vol. 19.

[20] J.S.W. Lamb and I. Melbourne, Arch. Ration. Mech. Anal. 149, 229 (1999).

[21] F. Marques, J.M. Lopez, and J. Shen, J. Fluid Mech. 455, 263 (2002).

[22] Y.A. Kuznetsov, Elements of Applied Bifurcation Theory, 2nd ed. (Springer, New York, 1998), Chap. 7.

[23] J.W. Swift and K. Wiesenfeld, Phys. Rev. Lett. 52, 705 (1984).

[24] S. Strogatz, Nonlinear Dynamics and Chaos (Addison-Wesley, Reading, MA, 1994).

[25] G. Pfister, A. Schulz, and B. Lensch, Eur. J. Mech. B/Fluids 10, 247 (1991).

[26] A.M. Rucklidge, Phys. Lett. A 284, 99 (2001). 